Revue internationale P.M.E.

Économie et gestion de la petite et moyenne entreprise

\title{
PME exportatrices et information en zones rurales ou zones urbaines
}

\author{
Pierre-André Julien, Robert Beaudoin et Ruphin Ndjambou
}

Volume 12, numéro 1-2, 1999

URI : https://id.erudit.org/iderudit/1008652ar

DOI : https://doi.org/10.7202/1008652ar

Aller au sommaire du numéro

\section{Éditeur(s)}

Presses de l'Université du Québec

ISSN

0776-5436 (imprimé)

1918-9699 (numérique)

Découvrir la revue

Citer cet article

Julien, P.-A., Beaudoin, R. \& Ndjambou, R. (1999). PME exportatrices et information en zones rurales ou zones urbaines. Revue internationale P.M.E., 12(1-2), 107-126. https://doi.org/10.7202/1008652ar

\section{Résumé de l'article}

La théorie économique traditionnelle soutient qu'il est plus difficile de se développer et d'exporter pour les PME en zones rurales là où les ressources, entre autres, informationnelles, sont plus rares qu'en zones urbaines en raison du manque d'économies d'agglomération. C'est ce que nous avons voulu vérifier à partir d'une enquête complexe auprès de PME manufacturières réparties sur le territoire du Québec et dont nous avons distingué 105 PME exportatrices oeuvrant dans des villes et 37 autres petites firmes rurales.

Les résultats montrent que les différences entre les PME exportatrices des deux zones sont minimes. Dans le cas des firmes rurales, elles se traduiront par une direction un peu moins instruite et expérimentée, un historique d'exportation plus jeune et donc une organisation moins complexe, une exportation moins " multinationale » et des produits un peu moins technologiques que ceux de leurs concurrents. Mais, en général, ni la localisation ni le niveau technologique des équipements semblent être des facteurs déterminants pour expliquer l'accès à l'information et la performance à l'exportation dans les deux zones. Il est donc possible de penser que l'évolution de l'environnement économique et des communications est en train d'annuler les différences qui distinguent le monde rural du monde urbain quant à la dynamique des PME ou du moins nous incite à rechercher d'autres différences pouvant expliquer les comportements des PME selon les localisations.
Ce document est protégé par la loi sur le droit d'auteur. L'utilisation des services d'Érudit (y compris la reproduction) est assujettie à sa politique d'utilisation que vous pouvez consulter en ligne.

https://apropos.erudit.org/fr/usagers/politique-dutilisation/ 


\title{
PME exportatrices et information en zones rurales ou zones urbaines*
}

Pierre-André JULIEN, Robert BEAUDOIN et Ruphin NDJAMBOU

Institut de recherche sur les PME

Université du Québec à Trois-Rivières

\section{MOTS CLÉS}

\section{PME exportatrices - Zones rurales et urbaines \\ Économies d'agglomération - Information et exportation \\ Information et performance}

\begin{abstract}
RÉSUMÉ
La théorie économique traditionnelle soutient qu'il est plus difficile de se développer et d'exporter pour les PME en zones rurales là où les ressources, entre autres, informationnelles, sont plus rares qu'en zones urbaines en raison du manque d'économies d'agglomération. C'est ce que nous avons voulu vérifier à partir d'une enquête complexe auprès de PME manufacturières réparties sur le territoire du
\end{abstract}

\section{LES AUTEURS}

Pierre-André Julien, Ph.D. en sciences économiques, est professeur en économie des PME à l'Université du Québec à Trois-Rivières et titulaire de la Chaire Bombardier Produits récréatifs en gestion du changement technologique dans les PME à l'Institut de recherche sur les PME. II a écrit seul ou avec d'autres plus de 70 articles, 200 rapports de recherche et 18 ouvrages, en prospective, en économie industrielle et en économie des PME. II est en outre consultant dans plusieurs institutions internationales dont l'OCDE.

Courriel: Pierre-andre_Julien@uqtr.uquebec.ca

Robert Beaudoin est détenteur d'un doctorat en gestion de l'Université Aix-Marseille III. Ses intérêts de recherche portent principalement sur l'étude des problèmes financiers des PME. II a œuvré pendant plusieurs années comme cadre supérieur à l'Université du Québec à Trois-Rivières. Il est membre du GREPME, depuis 1994, et directeur de l'Institut de recherche sur les PME, depuis 1997. Courriel: Robert_Beaudoin@uqtr.uquebec.ca

Ruphin Ndjambou est étudiant à la maîtrise en gestion des PME et assistant de recherche à la Chaire Bombardier à l'Université du Québec à Trois-Rivières.

Adresse : Chaire Bombardier, Institut de recherche sur les PME, Université du Québec à Trois-Rivières, 3351, boulevard des Forges, Trois-Rivières (Québec) G9A 5H7. Téléphone : (819) 376-5235. Télécopieur: (819) 376-5138. Courriel : Inrpme@uqtr.uquebec.ca

* Nous remercions les lecteurs pour leurs commentaires très constructifs. 
Québec et dont nous avons distingué 105 PME exportatrices œuvrant dans des villes et 37 autres petites firmes rurales.

Les résultats montrent que les différences entre les PME exportatrices des deux zones sont minimes. Dans le cas des firmes rurales, elles se traduiront par une direction un peu moins instruite et expérimentée, un historique d'exportation plus jeune et donc une organisation moins complexe, une exportation moins "multinationale » et des produits un peu moins technologiques que ceux de leurs concurrents. Mais, en général, ni la localisation ni le niveau technologique des équipements semblent être des facteurs déterminants pour expliquer l'accès à l'information et la performance à l'exportation dans les deux zones. Il est donc possible de penser que l'évolution de l'environnement économique et des communications est en train d'annuler les différences qui distinguent le monde rural du monde urbain quant à la dynamique des PME ou du moins nous incite à rechercher d'autres différences pouvant expliquer les comportements des PME selon les localisations.

\begin{abstract}
Traditional economic theory states that it is more arduous to develop and export for SMEs in rural zones. That is, external resources, such as information, are scarcer than in urban areas due to a lack of agglomeration economies. We assessed that statement in a complex study on 143 manufacturing SMEs from the province of Quebec, of which 105 were located in cities and 37 in rural areas.

The results reveal little differences between the two groups of enterprises. These are, rural businesses have slightly less experienced and educated management teams, briefer exporting track records, thus less complex export activities, narrower export spans, and products with somewhat lower technological levels compared to their competitors. However, neither location nor that variable combined to the technological level of equipment used were significant elements in explaining access to information and export performance in both territories. Therefore, it is conceivable that the evolution of our economic environment and of communications, could draw out the substantial differences between rural and urban areas pertaining to SMEs. These results single out the need of querying other dissimilarities which could elucidate the behaviours of SMEs from different regions.
\end{abstract}

\title{
RESUMEN
}

La teoría económica tradicional afirma que es más difícil para las PyMEs en medio rural desarrollarse y exportar, ya que los recursos externos y especialmente los de información, son más escasos que en las zonas urbanas, debido a la falta de economías de aglomeración. Esto es lo que hemos querido verificar a partir de una encuesta compleja realizada a PyMEs industriales repartidas sobre el territorio de Québec, trabajando con PyMEs 105 exportadoras de zonas urbanas y otras 37 pequeñas firmas de zonas rurales.

Los resultados muestran que las diferencias entre las PyMEs exportadoras de ambas zonas son mínimas. Estas son, para las firmas rurales, una dirección un poco menos instruída y experimentada, un histórico de exportación menor y una organización menos compleja, una exportación menos multinacional y productos con un menor componente tecnológico comparados a sus competidoras. Ahora bien, 
en términos generales, ni la localización ni esta variable ligada al nivel tecnológico de los equipos parecen ser los factores determinantes para explicar el acceso a la información y el éxito en la exportación en ambos territorios. Por ello, es posible pensar que la evolución del entorno económico y de las comunicaciones está anulando las diferencias entre el mundo rural y el urbano en lo que respecta a la dinámica de las PyMEs o, cuando menos, nos incita a buscar otras diferencias que puedan explicar los comportamientos según los territorios.

\section{ZUSAMMENFASSUNG}

Die traditionelle Betriebswirtschaftslehre besagt, dass KMU in ländlichen Zonen mehr Mühe bekunden, sich zu entwickeln und zu exportieren, weil externe Ressourcen (insbesondere Informationen) aufgrund fehlender Agglomerationseffekte nicht ausreichend vorhanden sind.

Diese Aussage sollte anhand einer ausführlichen Umfrage bei kleinen und mittleren Produktionsfirmen in und um Québec bestätigt werden. Dabei wurde unterschieden zwischen 105 exportierenden KMU, die in Städten tätig sind und 37 anderen kleinen ländlichen Firmen.

Die Resultate haben gezeigt, dass die Unterschiede zwischen den exportierenden KMU der beiden Zonen minim sind. Die städtischen Unternehmungen besitzen, wenn auch nur in geringem Ausmasse, besser ausgebildetere und erfahrenere Führungskräfte. Im weiteren weisen sie eine längere Vergangenheit im Bereich des Exports und aufgrund einer komplexeren Organisation, ein multinationaleres Exportwesen und technologisch leicht besser entwickelte Produkte im Vergleich zur Konkurrenz.

Generell gesehen hat aber weder der Standort noch das damit verbundene technologische Niveau der Einrichtungen einen bedeutenden Einfluss auf das Informationswesen und die Exportleistungen in den beiden Zonen.

Man könnte meinen, dass die Entwicklung des ökonomischen und kommunikativen Umfeldes die Unterschiede zwischen ländlichen und städtischen Zonen zu glätten beginnt, wenn man die Dynamik der KMU betrachtet. Zumindest regen diese Feststellungen an, nach anderen Unterschieden zu suchen, um standortspezifische Verhaltensweisen der KMU erklären zu können.

\section{Introduction}

À la différence des grandes entreprises qui, par définition, ont le plus souvent besoin d'un grand marché pour écouler leur forte production, marché qui dépasse les frontières nationales, la plupart des petites entreprises peuvent se contenter d'un marché national et même régional ou local. Et la mondialisation des marchés ne change pas cette situation pour un bon nombre d'entre elles qui profitent de leur proximité avec leur clientèle ou œuvrent sur des créneaux particuliers. Il existe toutefois un bon nombre d'autres PME qui non seulement font face à la concurrence internationale en raison du type de marché qu'elles couvrent, mais peuvent 
même profiter de l'abaissement des barrières douanières pour étendre ce marché (Julien, 1996). D'ailleurs, les gouvernements encouragent à l'aide de différents programmes spécifiques aux PME, ces comportements d'ouverture au marché international dans le but de soutenir la création d'emplois et d'aider à compenser les fluctuations de la conjoncture nationale. Malgré ces aides, le nombre de PME qui exportent, en particulier de façon systématique et bien organisée, est encore faible, entre $20 \%$ et $30 \%$ des petites entreprises manufacturières de plus de 10 employés (Julien et Morin, 1996). Une des barrières importantes à l'augmentation de ce pourcentage est la difficulté des petites entreprises à trouver les bonnes informations pour bien fonctionner sur des marchés souvent très différents du marché national (Seringhaus, 1987 ; Ali et Swiercz, 1991 ; Christensen, 1991). Ces informations particulières portent, par exemple, sur les différences de besoins des clients étrangers, sur les réseaux de distribution et les nouvelles formes de concurrence ou encore sur diverses ressources complémentaires nécessaires pour développer ces marchés, comme le courtage, les moyens de transport ou le financement particulier pour des transactions avec des étrangers. Ces difficultés d'obtenir l'information expliquent en partie pourquoi la plupart des PME qui commencent à exporter le font, d'abord, dans des marchés limitrophes ou dans des marchés à culture peu différente de celle du pays d'origine (OCDE, 1995). Lorsque les différences économiques et culturelles sont plus importantes, les PME utilisent divers moyens pour obtenir plus facilement ces informations comme les maisons de commerce, l'engagement d'un personnel d'origine du marché visé, la formation d'alliances avec des entreprises de ce marché (Marcotte et Julien, 1995) ou recourent à des firmes conseils spécialisées, nationales ou internationales, pour faciliter la pénétration du nouveau marché, du moins dans les premières années, puisque les études montrent que les PME exportatrices diminuent le recours à des ressources externes à mesure qu'elles prennent de l'expérience en exportation (Cavusgil, 1984 ; Chénier et Prince, 1990).

Pour leur part, ces ressources externes développent, elles aussi, leur expérience pour aider de nouvelles PME à exporter : c'est ainsi qu'à mesure qu'augmente le nombre de PME exportatrices, on voit apparaître de nouvelles maisons de commerce et autres firmes spécialisées sur certains marchés, aidées par les gouvernements. Ces ressources externes se retrouvent évidemment beaucoup plus facilement dans les métropoles ou dans les grandes villes, là où il y a plus de PME exportant ou ayant du potentiel pour l'exportation. En contrepartie, les PME des régions éloignées des grands centres, telles les régions rurales, ont plus de difficulté à avoir accès à ces ressources ou doivent passer par les grands centres pour les obtenir et pour finalement cumuler les informations clés pour réussir l'exportation.

Cette différence entre le nombre et la qualité des ressources entre les zones urbaines et celles rurales est d'ailleurs bien connue en économie régionale. On parle alors d'économies externes d'agglomération qui facilitent la connaissance et l'obtention des ressources à moindre coût pour les entreprises des milieux urbains, 
ce dont ne peuvent bénéficier les entreprises des régions rurales, notamment celles les plus éloignées. Toutefois, le développement des moyens de transport et des communications devrait réduire cet handicap pour les entreprises situées dans des milieux éloignés des grands centres urbains.

C'est ce que nous avons voulu vérifier à partir d'une enquête auprès d'un échantillon relativement représentatif de PME exportatrices manufacturières situées tant dans des régions rurales que dans des zones urbaines importantes. Les résultats, que nous rapportons dans cet article, montrent que si des différences notables sur le plan des ressources présentes, notamment informationnelles, existaient il y a quelques décennies, ces différences sont de plus en plus faibles, ou du moins les entreprises exportatrices rurales trouvent diverses façons pour pallier leur éloignement des villes. Nous exposerons, en premier lieu, les différences entre les deux types de localisation pour camper les hypothèses que nous voulons vérifier. En deuxième lieu, nous présenterons la méthodologie suivie et les analyses que nous avons faites. Nous discuterons par la suite des résultats obtenus pour terminer en signalant les limites de notre analyse.

\section{Zones urbaines et zones rurales}

Proulx (1991) note divers avantages des zones urbaines par rapport aux zones rurales quant aux ressources nécessaires pour exporter comme la concentration des services supérieurs financiers, de courtage, de transports et des réseaux facilitant les contacts pour mieux connaître les nouveaux marchés. Ces réseaux permettent de diminuer les coûts organisationnels, informationnels et technologiques pour adapter la production aux nouveaux besoins. Bailly et Boulianne (1992) citent la présence de chercheurs en R-D et de centres de valorisation pour adapter les produits, la qualité et la diversité des ressources complémentaires pour compléter les ressources internes, la variété de services spécialisés ou encore le nombre de maillages potentiels pour minimiser les risques et réduire les coûts de transaction. Tous ces facteurs sont résumés, entre autres, par Tellier (1993) ou Polèse (1994), qui parlent d'économies d'agglomération pour désigner l'ensemble des avantages que les entreprises peuvent tirer de leur regroupement dans un espace "préstructuré », occupé densément, généralement urbain.

Ces économies d'agglomération sont composées des deux éléments suivants :

- les économies de localisation liées à la production et constituant des avantages de long terme (proximité des sources d'approvisionnement, gains de productivité liés à la réduction des coûts de transport et d'information, maind'œuvre expérimentée, services spécialisés, innovation diffuse, etc.);

- les économies d'urbanisation dont profitent les entreprises (accès généralisé à toutes sortes d'information, clients et fournisseurs et équipementiers 
diversifiés, grande taille de marché pour le marché de base, accès aux infrastructures de qualité comme les autoroutes, les ports et les aéroports, accès aux services et aux programmes de l'administration publique, etc.).

En contrepartie, il y a des coûts d'agglomération comme le prix plus élevé du terrain et des immobilisations et autres taxes et redevances élevées, la congestion aux heures de pointe, et divers coûts de pollution (Mérenne-Schoumaker, 1991), entraînant d'autres coûts sociaux comme le stress et autres maladies urbaines.

Ces coûts sont souvent très inférieurs en zones rurales, ce qui représente des avantages. Ces zones, bien que ne jouissant pas des autres avantages de l'urbain, peuvent profiter, par exemple, d'un accès plus direct à diverses ressources naturelles (comme la forêt pour les scieries), de niveaux de salaires plus bas et de taxes foncières inférieures.

Bref, dans un cas ou dans l'autre, Goffette-Nagot et Schmitt (1998) relient ces avantages et désavantages à l'idée de proximité et d'interaction entre des ressources physiques et humaines nécessaires aux entreprises exportatrices qui diminueront ou, au contraire, augmenteront leurs coûts par rapport aux concurrents nationaux ou internationaux, ou encore à la proximité d'obtenir l'information nécessaire pour louer ou acheter ces ressources, pour mieux comprendre les marchés internationaux dans lesquels on veut investir et donc pour être plus en tant qu'exportateur. Cette proximité et ces interactions sont plus faibles dans les zones rurales que dans les zones urbaines, et augmenteraient les coûts des PME exportatrices rurales. Mais ces coûts peuvent être compensés en partie par une meilleure cohérence culturelle et sociale facilitant le partage d'information (Jayet, 1996), cohérence toutefois moins riche s'expliquant par une plus grande homogénéité des acteurs et probablement moins efficace sur le plan des relations avec les marchés internationaux. Ils peuvent en outre être annulés par les nouveaux moyens de communication informatisés abolissant les distances, dans plusieurs cas, et favorisant la formation de liens forts entre les acteurs des deux milieux ; ces liens peuvent parfois être renforcés par des complémentarités, comme on le voit dans les districts industriels (Pyke et Sengerberger, 1990) ou autres agglomérations d'entreprises à liens forts même dans des zones rurales (Arthur, 1990).

\section{Méthodologie}

Cette enquête avait d'abord pour but d'essayer de vérifier comment les PME avec différentes expériences d'exportation recherchaient et obtenaient leurs informations nécessaires pour réussir la pénétration des marchés étrangers et si certaines façons de faire permettaient d'être plus performants (Julien et al., 1998). C'est ce que nous avons entre autres voulu vérifier dans une enquête postale auprès de 3200 PME manufacturières de moins de 250 employés réparties dans six régions du Québec, 
soit le Bas-Saint-Laurent, Chaudière-Appalaches, Estrie, Laval, Mauricie-BoisFrancs et Montréal. Elle a été réalisée à partir de la banque de données du Centre de recherche industrielle du Québec (CRIQ) en ne retenant que les entreprises manufacturières qui disaient exporter : 346 entreprises ont retourné le questionnaire dûment rempli.

L'enquête avait été précédée d'une entrevue semi-structurée auprès de 12 entreprises exportatrices, ce qui nous a permis de préparer et de tester notre questionnaire. Celui-ci comprenait 58 questions portant sur les principales caractéristiques des entreprises, de la direction et du personnel, le type de production et le marché de ces entreprises, l'organisation et les caractéristiques d'exportation, le type et les sources d'informations recherchées et obtenues.

Nous avons retenu, pour cette étude, 39 questions traitant 1) de la localisation des entreprises, de leurs principales caractéristiques, notamment en dotation technologique, 2) des types d'informations recherchées, les sources utilisées et la fréquence de cette utilisation, et 3) des différents éléments pouvant permettre de mesurer la performance à l'exportation. L'information comprenait 21 types définis à partir d'éléments exposés par plusieurs auteurs (Samiee et Walters, 1990 ; Seringhaus, 1993) que nous avons divisés en cinq groupes : les informations sur les produits et sur le marché, sur la concurrence, sur les conditions d'opération sur le marché, sur les opportunités d'affaires et, enfin, sur le fonctionnement des exportations par les firmes. Nous avons relevé 28 sources d'information que nous avons regroupées selon leur provenance : les marchés et la distribution, l'industrie, les organismes à vocation commerciale, les programmes d'aides gouvernementaux et, enfin, les différents médias. Quant à la performance à l'exportation, nous nous sommes basés sur les critères proposés par Madsen (1987), soit 1) la part des exportations (en pourcentage du total des ventes), 2) la croissance des exportations aux États-Unis (en pourcentage), 3) la profitabilité des exportations par rapport aux ventes nationales et 4) la diversité des marchés (mesurée en pourcentage du marché desservi). Notons que notre référence au marché américain s'explique par la proximité et la taille de ce marché pour les PME québécoises qui y dirigent plus de $80 \%$ de leurs exportations.

Pour mesurer le niveau technologique de nos entreprises, nous nous sommes servis de la complexité des produits (échelle ordinale de 1 à 4), de la qualité des équipements par rapport à la concurrence (même échelle), de l'existence de la R-D (oui ou non), du recours à l'achat de brevets (idem) et de la situation de la firme au regard des normes de qualité (absente, en préparation, ou en cours d'implantation).

Parmi les entreprises ayant répondu au questionnaire, nous avons distingué 105 PME exportatrices implantées dans des villes et 37 autres petites firmes, en zone rurale. Pour les agglomérations urbaines, nous avons retenu les villes de plus de 40000 habitants selon le recensement de 1996. Quant aux zones rurales, nous 
nous sommes limités aux petites municipalités de moins de 5000 habitants et éloignées en moyenne de $46 \mathrm{~km}$ des grandes villes et de $26 \mathrm{~km}$ d'une autoroute. Nous avons laissé tomber les entreprises évoluant dans des zones intermédiaires, soit les banlieues des grandes villes ou les zones rurales proches de ces villes.

Le traitement des données a été basé, d'abord, sur des analyses statistiques de moyennes et de médianes pour décrire notre échantillon et, ensuite, sur des analyses de corrélations en utilisant les tests de Fisher et des tests d'Anova. Nous avons ainsi voulu vérifier les quatre hypothèses suivantes : 1) les PME exportatrices situées en zone urbaine accèdent plus facilement à l'information pour soutenir l'exportation que celles situées en zone rurale, soit notre variable indépendante; 2) les PME exportatrices urbaines performent mieux dans leurs exportations que leurs homologues rurales selon notre indice complexe (la variable dépendante); 3) les PME exportatrices urbaines sont mieux dotées sur le plan technologique que les autres, pour une variable de contrôle ; 4) cette dotation supérieure technologique influence positivement la capacité d'accès à l'information, ce qui peut réduire les effets de localisation sur la performance à l'exportation et boucler la boucle. Le graphique suivant illustre le cadre conceptuel avec les quatre hypothèses posées de même que la logique suivie.

\section{GRAPHIQUE 1}

\section{Cadre conceptuel résumant les hypothèses de recherche}

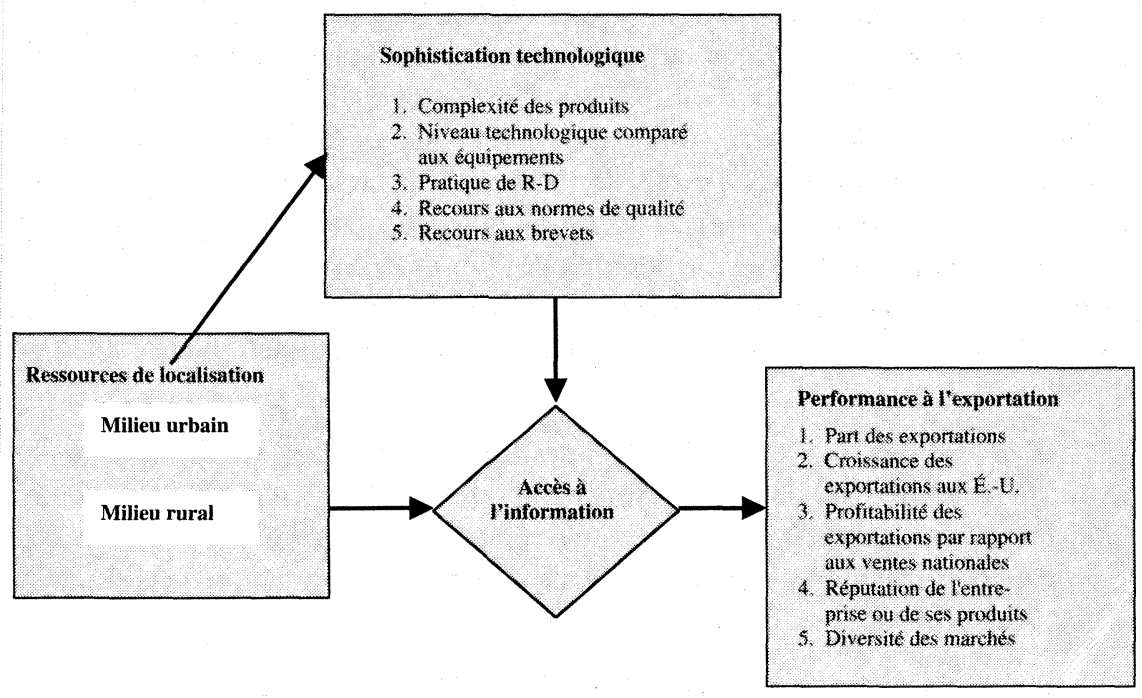




\section{Les résultats obtenus}

\subsection{Les caractéristiques des entreprises selon les deux types de milieux}

Comme le montre le tableau 1, les entreprises qui ont pris part à l'enquête ont en moyenne un effectif total de 48 employés pour les firmes urbaines et de 54 employés pour les rurales. De même, le chiffre d'affaires des PME rurales est un plus élevé que celui des PME urbaines, puisqu'un pourcentage plus élevé (49\% contre $44 \%$ ) ont un chiffre d'affaires de plus de 5 millions de dollars. Quant à l'évolution des ventes, les différences sont minimes dans le cas des firmes urbaines, $64 \%$ étant en croissance contre $65 \%$ dans le cas des PME rurales. Enfin, on trouve un peu plus de firmes rurales offrant des produits peu transformés.

En ce qui concerne le type de dirigeants de ces entreprises, nous relevons que le niveau de scolarisation n'est pas très différent et qu'il est relativement élevé, bien qu' on retrace un peu plus de dirigeants urbains ayant une formation universitaire. De même, ces derniers ont une expérience un peu plus forte dans leur entreprise. Enfin, le partage de leur temps entre les différentes fonctions de l'entreprise est relativement semblable : ces dirigeants consacrent du temps surtout dans les fonctions marketing et d'exportation en sus de la direction générale, fonctions relativement liées et généralement cruciales et plus complexes, surtout dans le cas de la fonction d'exportation.

Quant aux activités internationales, le tableau 2 montre des différences un peu plus grandes entre les PME urbaines et rurales, les premières ayant commencé à exporter plus tôt, elles exportent une part plus importante de leurs ventes et plus souvent hors l'Amérique du Nord. Ces trois variables sont d'ailleurs reliées puisque, selon le principe de l'apprentissage, les entreprises commencent à exporter en faible part sur des marchés plus proches pour ensuite augmenter cette part et s'introduire graduellement dans d'autres pays (Denis et Dépelteau, 1985 ; Gibiat,1993).

On peut également observer ce processus graduel dans l'évolution des ventes vers les régions plus éloignées de l'Ouest des États-Unis moins ouvertes aux PME rurales. Toutefois, les différences ne sont pas très grandes puisque ces dernières exportent une part plus grande de leurs produits dans les États de l'Est et du Centre américains, faute d'exporter autant dans les régions de l'Ouest et dans le reste du monde.

Ces faibles différences nous autorisent à penser que s'il y a des divergences importantes dans le recours à diverses sources d'information et dans l'utilisation de cette information, elles ne s'expliqueront pas par des différences importantes dans le type d'entreprise ou le type de dirigeant selon les deux localisations, sauf pour le niveau d'instruction qui pourrait jouer un rôle dans la capacité d'analyse 
Tableau 1

Caractéristiques générales des entreprises et des dirigeants selon les deux milieux

\begin{tabular}{|c|c|c|c|c|}
\hline \multirow[t]{2}{*}{ Variables analysées } & \multicolumn{2}{|c|}{$\begin{array}{l}\text { Firmes urbaines } \\
(\mathbf{N}=\mathbf{1 0 5})\end{array}$} & \multicolumn{2}{|c|}{$\begin{array}{l}\text { Firmes rurales } \\
\qquad(\mathbf{N}=37)\end{array}$} \\
\hline & Moyenne & Médiane & Moyenne & Médiane \\
\hline Nombre d'employés & 48 & 31 & 54 & 37 \\
\hline & Fréquence & $(\%)$ & Fréquence & $(\%)$ \\
\hline $\begin{array}{l}\text { Chiffre d'affaires en } 1996 \text { (en dollars) } \\
\text { - moins de } 0,5 \text { million } \\
\text { - de } 0,5 \text { à } 1 \text { million } \\
\text { - de } 1 \text { à } 5 \text { millions } \\
\text { - de } 5 \text { à } 10 \text { millions } \\
\text { - de } 10 \text { à } 20 \text { millions } \\
\text { - de } 20 \text { millions et plus }\end{array}$ & $\begin{array}{r}7 \\
11 \\
39 \\
17 \\
12 \\
17\end{array}$ & $\begin{array}{r}6,8 \\
10,7 \\
39,9 \\
16,5 \\
11,7 \\
16,5\end{array}$ & $\begin{array}{r}3 \\
2 \\
14 \\
12 \\
3 \\
3\end{array}$ & $\begin{array}{r}8,1 \\
5,4 \\
37,8 \\
32,4 \\
8,1 \\
8,1\end{array}$ \\
\hline $\begin{array}{l}\text { État de l'évolution des ventes } \\
\text { - stable } \\
\text { - en croissance } \\
\text { - à maturité }\end{array}$ & $\begin{array}{r}34 \\
84 \\
4\end{array}$ & $\begin{array}{r}32,4 \\
63,8 \\
3,8\end{array}$ & $\begin{array}{r}11 \\
24 \\
2\end{array}$ & $\begin{array}{r}29,7 \\
64,9 \\
5,4\end{array}$ \\
\hline $\begin{array}{l}\text { Types de produits vendus } \\
\text { - peu transformés } \\
\text { - semi-finis } \\
\text { - d'équipements industriels } \\
\text { - de consommation }\end{array}$ & $\begin{array}{r}2 \\
22 \\
4 \\
58\end{array}$ & $\begin{array}{r}1,9 \\
21,0 \\
22,9 \\
55,2\end{array}$ & $\begin{array}{r}2 \\
8 \\
9 \\
19\end{array}$ & $\begin{array}{r}5,4 \\
21,6 \\
24,3 \\
51,4\end{array}$ \\
\hline $\begin{array}{l}\text { Études complétées par le dirigeant } \\
\text { - primaire } \\
\text { - secondaire } \\
\text { - collégial } \\
\text { - universitaire }\end{array}$ & $\begin{array}{r}2 \\
17 \\
25 \\
59\end{array}$ & $\begin{array}{r}1,9 \\
16,5 \\
24,3 \\
57,3\end{array}$ & $\begin{array}{r}1 \\
9 \\
11 \\
15\end{array}$ & $\begin{array}{r}2,8 \\
25,0 \\
30,6 \\
41,7\end{array}$ \\
\hline $\begin{array}{l}\text { Fonctions autres que la direction générale } \\
\text { - aucune autre fonction } \\
\text { - marketing, vente } \\
\text { - exportation } \\
\text { - production } \\
\text { - achat, approvisionnement } \\
\text { - finance, comptabilité } \\
\text { - ressources humaines } \\
\text { - recherche et développement } \\
\end{array}$ & $\begin{array}{l}11 \\
64 \\
56 \\
24 \\
29 \\
46 \\
27 \\
34 \\
\end{array}$ & $\begin{array}{l}10,5 \\
61,0 \\
53,3 \\
22,9 \\
27,6 \\
43,8 \\
25,7 \\
32,4 \\
\end{array}$ & $\begin{array}{r}2 \\
23 \\
18 \\
12 \\
11 \\
10 \\
11 \\
14 \\
\end{array}$ & $\begin{array}{r}5,4 \\
62,2 \\
48,6 \\
32,4 \\
29,7 \\
27,0 \\
29,7 \\
37,8 \\
\end{array}$ \\
\hline & Moyenne & Médiane & Moyenne & Médiane \\
\hline $\begin{array}{l}\text { Expérience dans l'entreprise } \\
\text { (en nombre d'années) }\end{array}$ & 14 & 11 & 10 & 9 \\
\hline
\end{tabular}

de l'information. Qu'en est-il, par ailleurs, des types d'information et des sources utilisées, éléments fondamentaux de cette recherche ? Les tableaux 3 et 4 révèlent que, là aussi, les différences ne sont pas très grandes. 
TABLEAU 2

Caractéristiques générales des activités exportatrices selon les deux milieux

\begin{tabular}{lcccc}
\hline Variables analysées & \multicolumn{2}{c}{$\begin{array}{c}\text { Firmes urbaines } \\
(\mathbf{N = 1 0 5 )}\end{array}$} & \multicolumn{2}{c}{$\begin{array}{c}\text { Firmes rurales } \\
\mathbf{N}=\mathbf{3 7 )}\end{array}$} \\
\hline & Fréquence & $\mathbf{( \% )}$ & Fréquence & $\mathbf{( \% )}$ \\
\hline Entreprises desservant les marchés & & & & \\
- Est des États-Unis & 78 & 84,6 & 28 & 87,5 \\
- Sud des États-Unis & 55 & 59,8 & 14 & 43,8 \\
- Centre des États-Unis & 52 & 56,5 & 19 & 59,4 \\
- Ouest des États-Unis & 46 & 50,0 & 12 & 37,5 \\
\hline & Moyenne & Médiane & Moyenne & Médiane \\
\hline Répartition des ventes en 1996 & & & & \\
- Québec & 45,6 & 40,0 & 39,7 & 35,0 \\
- reste du Canada & 18,9 & 10,0 & 19,7 & 15,0 \\
- États-Unis & 25,6 & 12,5 & 35,0 & 30,0 \\
- ailleurs dans le monde & 9,8 & 2,9 & 5,6 & 0,0 \\
\hline Croissance des ventes en trois ans & & & & \\
- Québec et reste du Canada & $1,49 \%$ & & $1,82 \%$ & \\
- États-Unis & $2,24 \%$ & & $2,12 \%$ & \\
- ailleurs dans le monde & $2,29 \%$ & & $1,59 \%$ & \\
\hline Date du début des ventes & & & & \\
- Québec et reste du Canada & 1978 & 1984 & 1984 & 1985 \\
- États-Unis & 1986 & 1989 & 1988 & 1988 \\
- ailleurs dans le monde & 1989 & 1993 & 1991 & 1991 \\
\hline
\end{tabular}

Dans le premier tableau, nous indiquons l'importance que les répondants attachaient à une vingtaine de types d'information rapportés dans la littérature en ce qui concerne les marchés et les produits, la concurrence, les conditions d'opérations sur les marchés, les occasions d'affaires et le fonctionnement des exportations. Dans les deux milieux, les firmes accordent avant tout de l'importance aux informations sur les prix pratiqués sur les marchés, sur le potentiel ou l'adaptation des produits (les firmes rurales donnant un peu moins d'importance à ces éléments), les délais de livraison quant aux conditions d'opération sur les marchés, les forces et faiblesses des concurrents et, enfin, sur les nouveaux produits et les goûts ou besoins des clients. Les informations sur les opérations douanières, le transport, les aspects légaux et l'aide gouvernementale revêtent le moins d'importance dans les deux cas.

Au tableau 4, nous avons analysé la fréquence des contacts que les deux groupes de PME exportatrices entretenaient avec 28 types de sources d'information aux fins des exportations. Ces sources sont classées en cinq catégories se rapportant au marché et à la distribution, à l'industrie, à des organisations à vocation commerciale, à des institutions d'aides et à des sources écrites et aux médias. Encore une fois, la fréquence de recours aux sources d'information est relativement similaire 
TABLEAU 3

Importance des informations pour l'exportation dans les deux milieux

\begin{tabular}{|c|c|c|c|c|}
\hline & \multicolumn{2}{|c|}{$\begin{array}{l}\text { Firmes urbaines } \\
\qquad(\mathbf{N}=\mathbf{1 0 5})\end{array}$} & \multicolumn{2}{|c|}{$\begin{array}{l}\text { Firmes rurales } \\
\qquad(\mathbf{N}=\mathbf{3 7})\end{array}$} \\
\hline & $\begin{array}{l}\text { Moyenne } \\
\text { (médiane)* }\end{array}$ & Rang & $\begin{array}{c}\text { Moyenne } \\
\text { (médiane)* }\end{array}$ & Rang \\
\hline $\begin{array}{l}\text { Marchés et produits } \\
-\quad \text { prix sur les marchés } \\
-\quad \text { potentiel / adaptation des produits } \\
-\quad \text { nouveaux produits } \\
-\quad \text { goûts / besoins des clients } \\
-\quad \text { informations générales sur le marché } \\
-\quad \text { méthodes de promotion }\end{array}$ & $\begin{array}{l}2,28(2) \\
2,25(2) \\
2,04(2) \\
2,01(2) \\
1,91(2) \\
1,66(2)\end{array}$ & $\begin{array}{r}1 \\
2 \\
5 \\
6 \\
10 \\
13\end{array}$ & $\begin{array}{l}2,32(3) \\
2,07(2) \\
2,09(2) \\
1,89(2) \\
1,89(2) \\
1,67(1)\end{array}$ & $\begin{array}{r}1 \\
5 \\
2 \\
9 \\
10 \\
11\end{array}$ \\
\hline $\begin{array}{l}\text { Concurrence } \\
-\quad \text { forces et faiblesses des concurrents } \\
\text { - } \quad \text { stratégie des concurrents }\end{array}$ & $\begin{array}{l}2,06(2) \\
1,91(2)\end{array}$ & $\begin{array}{l}4 \\
9 \\
\end{array}$ & $\begin{array}{l}2,07(2) \\
1,95(2)\end{array}$ & $\begin{array}{l}6 \\
8\end{array}$ \\
\hline $\begin{array}{l}\text { Conditions d'opération sur les marchés } \\
\text { - délais de livraison / service } \\
\text { - normes techniques, réglementations } \\
\text { - licences d'import / export }\end{array}$ & $\begin{array}{l}2,20(2) \\
1,84(1) \\
1,21(1)\end{array}$ & $\begin{array}{r}3 \\
12 \\
20\end{array}$ & $\begin{array}{l}2,09(2) \\
1,67(2) \\
1,28(1)\end{array}$ & $\begin{array}{r}3 \\
12 \\
20\end{array}$ \\
\hline $\begin{array}{l}\text { Occasions d'affaires } \\
\text { - clients potentiels } \\
\text { - contacts et relations avec le milieu } \\
\text { - } \text { possibilités de partenariat }\end{array}$ & $\begin{array}{l}1,99(2) \\
1,97(2) \\
1,18(1)\end{array}$ & $\begin{array}{r}7 \\
8 \\
21\end{array}$ & $\begin{array}{l}2,08(2) \\
1,98(2) \\
1,16(1)\end{array}$ & $\begin{array}{r}4 \\
7 \\
21\end{array}$ \\
\hline $\begin{array}{l}\text { Fonctionnement des exportations } \\
\text { - modes de paiement, contrats } \\
\text { - } \text { transport } \\
\text { - amélioration / développement } \\
\text { de la distribution } \\
\text { - } \text { commission / marges à accorder } \\
\text { - opérations douanières } \\
\text { - } \\
\text { - aspects légaux du commerce international } \\
\text { aide gouvernementale }\end{array}$ & $\begin{array}{r}1,40(1) \\
1,54(2) \\
1,45(1) \\
\text { al } 1,40(1) \\
1,42(1)\end{array}$ & $\begin{array}{l}19 \\
15 \\
16 \\
18 \\
17\end{array}$ & $\begin{array}{l}1,57(2) \\
1,40(1) \\
1,43(1) \\
1,42(1) \\
1,44(1)\end{array}$ & $\begin{array}{l}15 \\
19 \\
17 \\
18 \\
16\end{array}$ \\
\hline
\end{tabular}

Note : Dans certains cas, il y a un écart par rapport à $100 \%$ qui résulte des omissions de réponse.

* Échelle ordinale allant de 0 (pas important) à 4 (très important). Les chiffres sont arrondis à l'unité.

dans les deux groupes de firmes. Les sources les plus fréquemment utilisées par l'ensemble des firmes sont celles qui se rapportent au marché et à la distribution avec une médiane de 2 . Il s'agit plus précisément des clients et représentants, et des agents, grossistes et distributeurs. À l'inverse, les sources les moins fréquemment utilisées comprennent non seulement les organisations à vocation commerciale, incluant les maisons et associations de commerce et les agences de marketing, mais aussi les organismes d' aide comme les organismes gouvernementaux et de normalisation, les consultants et les intervenants locaux. 
TABleau 4

Utilisation des sources d'information pour l'exportation dans les deux milieux

\begin{tabular}{|c|c|c|c|c|}
\hline & \multicolumn{2}{|c|}{$\begin{array}{l}\text { Firmes urbaines } \\
\qquad(\mathbf{N}=\mathbf{1 0 5})\end{array}$} & \multicolumn{2}{|c|}{$\begin{array}{c}\text { Firmes rurales } \\
\quad(\mathbf{N}=\mathbf{3 7})\end{array}$} \\
\hline & $\begin{array}{l}\text { Moyenne } \\
\text { (médiane)* }\end{array}$ & Rang & $\begin{array}{l}\text { Moyenne } \\
\text { (médiane)* }\end{array}$ & Rang \\
\hline $\begin{array}{l}\text { Marchés et distribution } \\
\text { - clients } \\
\text { - représentants } \\
\text { - agents / grossistes / distributeurs } \\
\text { - bureaux de vente } \\
\text { - } \text { filiale / compagnie - mère }\end{array}$ & $\begin{array}{l}2,17(2) \\
2,08(2) \\
1,69(2) \\
1,08(0) \\
0,74(0)\end{array}$ & $\begin{array}{r}1 \\
2 \\
4 \\
10 \\
15\end{array}$ & $\begin{array}{l}2,11(2) \\
2,09(3) \\
1,86(2) \\
0,72(0) \\
0,52(0)\end{array}$ & $\begin{array}{r}1 \\
2 \\
3 \\
16 \\
22\end{array}$ \\
\hline $\begin{array}{l}\text { Industrie } \\
\text { - } \quad \text { fournisseurs } \\
\text { - } \text { autres entreprises / relations } \\
\text { - entreprise - partenaire } \\
\text { - } \text { concurrents } \\
\text { - } \text { regroupements d'entreprises / } \\
\quad \text { gens d'affaires } \\
\text { - } \text { association sectorielles }\end{array}$ & $\begin{array}{l}1,71(2) \\
1,10(1) \\
0,68(0) \\
0,72(1)\end{array}$ & $\begin{array}{r}3 \\
9 \\
18 \\
16\end{array}$ & $\begin{array}{l}1,61(2) \\
1,00(1) \\
0,62(0) \\
0,84(1)\end{array}$ & $\begin{array}{r}4 \\
9 \\
19 \\
13\end{array}$ \\
\hline $\begin{array}{l}\text { Organisation à vocation commerciale } \\
\text { - foires à l'étranger } \\
\text { - } \text { foires au Canada } \\
\text { - maisons / Assoc. commerciales } \\
\text { au Canada } \\
\text { - maisons / Assoc. commerciales } \\
\quad \text { à l'étranger } \\
\text { - agences marketing au Canada } \\
\text { - } \text { agences marketing à l'étranger }\end{array}$ & $\begin{array}{l}1,25(1) \\
1,2291) \\
0,47(0)\end{array}$ & $\begin{array}{l}25 \\
26 \\
27 \\
28 \\
\end{array}$ & $\begin{array}{l}1,42(1) \\
1,28(1) \\
0,27(0) \\
0,21(0) \\
0,15(0) \\
0,10(0)\end{array}$ & $\begin{array}{l}24 \\
26 \\
27 \\
28\end{array}$ \\
\hline $\begin{array}{l}\text { Organismes d'aides } \\
\text { - } \text { institutions financières } \\
\text { - } \text { organismes gouvernementaux } \\
\text { au Canada } \\
\text { - } \text { intervenants locaux } \\
\text { - } \text { organismes gouvernementaux } \\
\text { à l'étranger } \\
\text { - consultants au Canada } \\
\text { - } \text { agences / organismes de normalisation } \\
\text { - consultants à l'étranger }\end{array}$ & $\begin{array}{c}0,6590) \\
0,58(0) \\
0,52(0) \\
0,50(0)\end{array}$ & $\begin{array}{l}20 \\
22 \\
23 \\
24\end{array}$ & $\begin{array}{l}0,9191) \\
0,73(1) \\
\\
0,35(0) \\
0,53(0) \\
0,55(0) \\
0,27(0)\end{array}$ & $\begin{array}{l}23 \\
21 \\
20 \\
25\end{array}$ \\
\hline $\begin{array}{l}\text { Sources écrites et médias: } \\
-\quad \text { revues d'affaires spécialisées } \\
\text { - Internet } \\
-\quad \text { journaux, télévision, radio } \\
-\quad \text { données statistiques }\end{array}$ & $\begin{array}{c}1,66(2) \\
1,01(1) \\
0,84(1) \\
0,88(1)\end{array}$ & $\begin{array}{c}5 \\
11 \\
14 \\
13\end{array}$ & $\begin{array}{l}1,43(1) \\
0,93(1) \\
0,85(1) \\
0,74(0)\end{array}$ & $\begin{array}{r}5 \\
10 \\
12 \\
14\end{array}$ \\
\hline
\end{tabular}

Note : Dans certains cas, il y a un écart par rapport à $100 \%$ qui résulte des omissions de réponse.

* Échelle ordinale allant de 0 (jamais en contact) à 4 (toujours en contact). Les chiffres sont arrondis à l'unité. 
Ces résultats semblent correspondre à ceux des études portant sur la veille montrant que les sources les plus utilisées sont les sources personnelles d'affaires plus ou moins informelles (Julien, 1995) ou encore sur la distinction que fait Léo (1993) entre informations de fonctionnement et informations de développement.

De l'analyse de ces sources, se dégagent trois grandes caractéristiques. Premièrement, les deux groupes de firmes ont une faible perception de l'utilité des organismes gouvernementaux : c'est ce qui ressort de la majorité des travaux sur les PME exportatrices et même non exportatrices. Deuxièmement, elles affichent toutes une ferme volonté de recourir aux ressources externes même si c'est encore dans une faible proportion. Troisièmement, les firmes rurales recourent plus aux foires que les firmes urbaines. Mais dans l'ensemble, la localisation, qu'elle soit rurale ou urbaine, n'affecte pas l'accès à l'information par les PME exportatrices.

\subsection{Analyses statistiques}

En utilisant des tests d'Anova pour vérifier nos hypothèses, nous avons relevé que les différences entre les PME exportatrices des deux milieux sont minimes. Commençons par la première hypothèse, à savoir que les PME exportatrices de milieu urbain accéderaient plus facilement à l'information que celles de milieu rural. Le tableau 5 montre qu'un seul type de sources d'information est considéré comme plus important et se révèle plus utilisé par les PME urbaines. Pour toutes les autres sources, les différences ne sont pas significatives.

L'hypothèse 2 posait que les PME exportatrices urbaines performaient mieux à l'exportation que les PME rurales. Au tableau 6, nous voyons encore ici qu'il n'existe pas de différences significatives $(\mathrm{p}>0,05)$ dans la performance des firmes exportatrices rurales et urbaines selon les critères retenus. En effet, les résultats détaillés indiquent que les firmes urbaines exportent en moyenne pour $35,4 \%$ de leur production et ont connu une croissance des exportations aux États-Unis en moyenne de 10 à $19 \%$; ces entreprises ont une bonne réputation (avec une moyenne de 4,17 sur une échelle de 5) et diversifient leurs marchés dans une proportion moyenne de 3,34 sur une échelle de 4. Ces résultats sont sensiblement les mêmes (ou plus élevés, dans certains cas) chez les firmes rurales, soit respectivement des moyennes de $40,6 \%$ et de 10 à $19 \%$, un niveau de réputation de 4,19 et de diversification de 2,91. Cette hypothèse est donc infirmée.

Dans notre troisième hypothèse, nous avons avancé l'idée que la proximité des grands centres d'affaires et de recherche pouvait avantager les PME exportatrices urbaines sur le plan technologique. Au tableau 7 , on peut voir à nouveau que les différences sont très faibles sauf dans les cas du recours aux brevets et de l'implantation des normes de qualité; et, dans ces cas, les différences ne sont pas significatives. Les deux groupes des firmes échantillonnées ont donc un niveau technologique relativement comparable. 


\section{TABLEAU 5}

Différences dans la perception de l'importance des informations et de l'utilisation des sources d'information selon la localisation

\begin{tabular}{lllc}
\hline Variables analysées & \multicolumn{2}{c}{$\begin{array}{l}\text { Moyennes selon } \\
\text { la localisation }\end{array}$} & \multicolumn{1}{c}{$\begin{array}{c}\text { F } \\
\text { ANOVA }\end{array}$} \\
\hline & Urbain & Rural & \\
\hline Types d'information & & & \\
- information sur les marchés et les produits & 2,02 & 1,98 & 0,06 \\
- information sur la concurrence & 1,97 & 2,01 & 0,48 \\
- information sur les conditions d'opération & & & \\
- sur les marchés & 1,72 & 1,68 & 0,72 \\
- information sur les occasions d'affaires & 1,66 & 1,74 & 0,20 \\
- information sur le fonctionnement des exportations & 1,52 & 1,49 & 0,04 \\
\hline Sources d'information & & & \\
- sources se rapportant au marché et à la distribution & 1,54 & 1,42 & 0,71 \\
- sources se rapportant à l'industrie & 0,91 & 0,93 & 0,06 \\
- sources touchant les organisations commerciales & 0,42 & 0,27 & $\mathbf{3 , 2 0}$ \\
- sources sur les aides diverses & 0,93 & 0,90 & 0,06 \\
- sources écrites (revues et autres médias) & 1,09 & 0,99 & 1,02 \\
\hline
\end{tabular}

a) Échelle ordinale allant de 0 (pas important) à 4 (très important).

b) Échelle ordinale allant de 0 (jamais en contact) à 4 (toujours en contact). Le chiffres sont arrondis à l'unité.

* $\mathrm{p} \leq 0,10 * * \mathrm{p} \leq 0,05 * * * \mathrm{p} \leq 0,01 * * * * \mathrm{p} \leq 0,001$

\section{Tableau 6}

Différences dans la performance à l'exportation selon la localisation

\begin{tabular}{|c|c|c|c|}
\hline \multirow[t]{2}{*}{ Indicateurs de performance } & \multicolumn{2}{|c|}{$\begin{array}{l}\text { Moyennes selon } \\
\text { la localisation }\end{array}$} & \multirow[t]{2}{*}{$\begin{array}{c}\text { F } \\
\text { ANOVA }\end{array}$} \\
\hline & Urbain & Rural & \\
\hline Part des ventes exportées & $35,4 \%$ & $40,6 \%$ & 0,69 \\
\hline Croissance des ventes aux États-Unis & $(10$ à $19 \%)$ & $(10$ à $19 \%)$ & 0,15 \\
\hline Profitabilité des exportations ${ }^{\mathrm{a}}$ & 2,58 & 2,61 & 0,02 \\
\hline Réputation de l'entreprise & & & \\
\hline sur les marchés étrangers ${ }^{b}$ & 4,17 & 4,19 & 0,01 \\
\hline Diversification des marchés étrangers & 3,34 & 2,91 & 2,38 \\
\hline
\end{tabular}

a) Échelle allant de 1 (moins profitables) à 4 (beaucoup plus profitables).

b) Échelle allant de 1 (peu connue en dehors de la région) à 5 (très connue et réputée dans le monde).

$* \mathrm{p} \leq 0,10 * * \mathrm{p} \leq 0,05 * * * \mathrm{p} \leq 0,01 * * * * \mathrm{p} \leq 0,001$

Enfin, pour vérifier la quatrième hypothèse, soit que la modernisation technologique influence positivement l'accès à l'information de sorte qu'elle réduit l'impact de la localisation sur la performance à l'exportation, nous avons d'abord divisé notre échantillon en deux groupes selon le niveau technologique : les firmes 
peu dotées (groupe 1:57 entreprises) et les fortement dotées (groupe 2: 82 entreprises). Au tableau 8 , où nous avons les firmes peu dotées technologiquement parlant, on ne voit pas plus de différences selon la localisation sauf dans le cas de la source provenant des organisations commerciales où la différence est significative mais faible, avec une probabilité de $\mathrm{p} \leq 0,10$ seulement. Pour le reste, le recours aux différents types et aux diverses sources d'information ainsi que la performance exportatrice sont semblables quelle que soit la localisation de ces entreprises.

Quant aux firmes beaucoup plus technologiques, nous pouvons constater au tableau 9 que la situation est passablement similaire puisque tant les firmes urbaines que rurales recourent à peu près aux mêmes types d'information et utilisent les mêmes sources; de plus, leur performance à l'exportation est semblable : toutes les 15 dimensions sont non corrélées $(\mathrm{p}>0,05)$. Ainsi, la deuxième conclusion qui ressort de ces résultats, dans le cas des PME fortement dotées sur le plan technologique, est la même que la première, car il n'y a aucune différence significative au regard de l'accès à l'information et de la performance à l'exportation que ces entreprises soient localisées en zone urbaine ou en zone rurale. Bref, ni la localisation, ni la dotation technologique ne semblent être des facteurs déterminants pour expliquer l'accès à l'information et la performance à l'exportation des PME exportatrices urbaines ou rurales.

\section{Conclusion}

Ainsi, il nous faut rechercher l'explication de la capacité des firmes rurales à accéder à l'information au même titre que leurs homologues urbaines et de leur performance à l'exportation ailleurs que dans la localisation et la technologie. À moins de considérer que la définition que nous avons utilisée pour distinguer les deux localisations n'est pas suffisamment précise notamment pour tenir compte du fait que les PME exportatrices sont souvent les plus dynamiques et que leurs comportements diffèrent beaucoup des autres entreprises en particulier pour cet aspect de recherche de l'information, compte tenu de leurs besoins particuliers. D'ailleurs, certains chercheurs comme Goffette-Nagot et Schmitt (1998) rappellent que, par exemple, le concept de localisation et de ses différences est beaucoup plus complexe que celui que nous avons pu évoquer dans notre analyse. Au-delà de la proximité physique et des économies d'agglomération, poursuivent ces auteurs, il peut s'appliquer entre autres aux proximités organisationnelles ou institutionnelles. C'est d'autant plus vrai que l'information que l'on obtient est une ressource intangible et donc facilement transportable. Elle est variable, selon la relation entre informateur et informé, périssable, demandant une transmission rapide, cumulative et complexe pour passer du savoir au savoir-faire et à l'action. Elle peut être obtenue de diverses façons par des moyens de communication électroniques ou à l'occasion de toutes sortes de déplacements. Par exemple, il n'est pas sûr que les entrepreneurs ruraux aient moins de contacts 


\section{TABLEAU 7}

Différences de niveau technologique selon la localisation

\begin{tabular}{lccc}
\hline Niveau technologique & \multicolumn{2}{c}{$\begin{array}{c}\text { Moyennes selon } \\
\text { la localisation }\end{array}$} & \multicolumn{2}{c}{$\begin{array}{c}\text { F } \\
\text { ANOVA }\end{array}$} \\
\hline & Urbain & Rural & \\
\hline Complexité des produits $^{\mathrm{a}}$ & 3,35 & 3,30 & 0,03 \\
Niveau technologique comparéb $^{\mathrm{b}}$ & 2,42 & 2,59 & 1,25 \\
Recherche et développement $_{\text {Recours aux brevets }}$ & $86 \%$ & $86 \%$ & 0,0007 \\
Implantation des normes de qualité & $16 \%$ & $10 \%$ & 0,62 \\
& $44 \%$ & $37 \%$ & 0,43 \\
\hline
\end{tabular}

a) Échelle ordinale allant de 1 (production de pièces simples) à 4 (production de pièces très spécialisées).

b) Échelle ordinale allant de 1 (moins avancé) à 4 (très en avance).

$* \mathrm{p} \leq 0,10 * * \mathrm{p} \leq 0,05 * * * \mathrm{p} \leq 0,01 * * * * \mathrm{p} \leq 0,001$

TABLEAU 8

Vérification de l'hypothèse 4

pour les firmes peu dotées sur le plan technologique

(Groupe 1 - N = 57 entreprises)

\begin{tabular}{lccc}
\hline Variables analysées & \multicolumn{2}{c}{$\begin{array}{c}\text { Moyennes selon } \\
\text { la localisation }\end{array}$} & $\begin{array}{c}\text { F } \\
\text { ANOVA }\end{array}$ \\
\hline & Urbain & \multicolumn{1}{c}{ Rural } & \\
\hline & & & \\
\hline Types d'information & 2,02 & 1,96 & 0,10 \\
- information sur les marchés et les produits & 1,98 & 1,90 & 0,14 \\
- information sur la concurrence & & & \\
- information sur les conditions d'opération & 1,79 & 1,63 & 0,56 \\
sur les marchés & 1,68 & 1,60 & 0,10 \\
- information sur les occasions d'affaires & 1,65 & 1,54 & 0,27 \\
- information sur le fonctionnement des exportations & & & \\
Sources d'information & 1,32 & 1,47 & 0,62 \\
- sources se rapportant au marché et à la distribution & 0,87 & 0,80 & 0,25 \\
- sources se rapportant à l'industrie & 0,40 & 0,19 & $3,22 *$ \\
- sources touchant les organisations commerciales & 0,85 & 0,72 & 0,99 \\
- sources sur les aides diverses & 1,04 & 0,84 & 1,19 \\
- sources écrites (revues et autres médias) & & & \\
Performance à l'exportation & $25,9 \%$ & $34,4 \%$ & 1,41 \\
- part des ventes exportées & $(10$ à $19 \%)$ & $(10$ à $19 \%)$ & 0,12 \\
- croissance des ventes aux États-Unis & 2,44 & 2,55 & 0,12 \\
- profitabilité des exportations & & & \\
- réputation de l'entreprise & 3,81 & 3,83 & 0,004 \\
sur les marchés étrangers & 2,86 & 2,31 & 2,64 \\
\hline - diversification des marchés étrangers & & &
\end{tabular}

$* \mathrm{p} \leq 0,10 * * \mathrm{p} \leq 0,05 * * * \mathrm{p} \leq 0,01 * * * * \mathrm{p} \leq 0,001$ 


\section{TABLEAU 9}

Vérification de l'hypothèse 4 pour les firmes fortement dotées sur le plan technologique (Groupe $2-\mathbf{N}=82$ entreprises)

\begin{tabular}{llcc}
\hline Variables analysées & \multicolumn{2}{c}{$\begin{array}{c}\text { Moyennes selon } \\
\text { la localisation }\end{array}$} & $\begin{array}{c}\text { F } \\
\text { ANOVA }\end{array}$ \\
\hline & Urbain & Rural & \\
\hline & & & \\
Types d'information & 2,02 & 2,01 & 0,001 \\
- information sur les marchés et les produits & 1,97 & 2,13 & 0,45 \\
- information sur la concurrence & & & \\
- information sur les conditions d'opération & 1,68 & 1,62 & 0,04 \\
- sur les marchés & 1,65 & 1,88 & 0,99 \\
- information sur les opportunités d'affaires & 1,44 & 1,44 & 0,00 \\
- information sur le fonctionnement des exportations & & & \\
Sources d'information & 1,67 & 1,38 & 2,14 \\
- sources se rapportant au marché et à la distribution & 0,93 & 1,07 & 1,02 \\
- sources se rapportant à l'industrie & 0,43 & 0,36 & 0,31 \\
- sources touchant les organisations commerciales & 0,97 & 1,10 & 0,68 \\
- sources sur les aides diverses & 1,17 & 1,15 & 0,007 \\
- sources écrites (revues et autres médias) & & & \\
\hline Performance à l'exportation & $41,5 \%$ & $47,6 \%$ & 0,43 \\
- part des ventes exportées & $(10$ à $19 \%)$ & $(10$ à $19 \%)$ & 0,70 \\
- croissance des ventes aux États-Unis & 2,66 & 2,68 & 0,005 \\
- profitabilité des exportations & 4,37 & 4,55 & 0,59 \\
- réputation de l'entreprise sur les marchés étrangers & 3,62 & 3,58 & 0,008 \\
\hline - diversification des marchés étrangers & & & \\
\hline
\end{tabular}

$* \mathrm{p} \leq 0,10 * * \mathrm{p} \leq 0,05 * * * \mathrm{p} \leq 0,01 * * * * \mathrm{p} \leq 0,001$

avec leurs clients que les entrepreneurs urbains. En effet, les deux doivent se déplacer, même si les premiers peuvent avoir à assumer des coûts supplémentaires pour transiter par la ville avant de rejoindre les marchés étrangers.

Ajoutons que notre distinction entre zones urbaines et rurales au Québec aurait peut-être dû considérer trois localisations, soit la métropole (Montréal), les villes intermédiaires et le monde rural. Sur le plan de l'exportation, il est possible que seules les villes internationales, donc ouvertes sur le monde, offrent de réels avantages en matière de performance à l'exportation et d'accès à l'information internationale.

De même, les critères que nous avons retenus pour définir la performance du point de vue de l'exportation sont discutables. D'autres critères sont cités par les chercheurs (Savoie, 1993 ; OCDE, 1995), soit le volume des ventes, la productivité, la qualité des décisions, la qualité des produits et du service à la clientèle, le chiffre d'affaires, le nombre de personnes impliquées dans l'exportation. Il est possible que des critères différents auraient donné des résultats différents. Enfin, nous avons mesuré le niveau technologique des entreprises échantillonnées par des critères qui ne font pas référence aux nouvelles technologies de l'information et 
de la communication ; nous aurions très bien pu tenir compte de ces nouvelles technologies dans l'analyse de l'impact de la modernisation technologique sur l'accès à l'information et la performance à l'exportation.

Notre analyse demeure tout de même intéressante, car elle remet en question les notions traditionnelles de localisation urbaine et rurale ainsi que celles des avantages presque certains des économies d'agglomération. Avec les changements économiques actuels où émergent de nouveaux besoins de connaissance et se multiplient les technologies d'information, il est possible que ces vieilles distinctions soient en train de s'estomper. Du moins, une telle analyse peut aider à nourrir les discussions au sujet de la localisation.

\section{Bibliographie}

ALI, A. et R.M. SwIERCZ (1991), «Firm size and export behavior : lessons from the Midwest », Journal of Small Business Management, vol. 29, $\mathrm{n}^{\circ}$ 2, p. 71-78.

ARTHUR, W.B. (1990), «Silicon Valley' cluster: when do increasing returns imply monopoly?», Mathematical Social Science, $\mathrm{n}^{\circ} 19$, p. 235-251.

BAILly, A. et L. BoulianNe (1992), «Les nouveaux réseaux métropolitains : théories, études de cas et comparaison internationales », Revue Canadienne des Sciences Régionales, vol. 15, $\mathrm{n}^{\circ} 3$, automne, p. 361-376.

CAVusgil, S.T. (1984), «Differences among exporting firms based on their degree of internationalization », Journal of Business Research, vol. 12, $\mathrm{n}^{\circ}$ 2, p. 195-208.

CHÉNIER, J. et M. PRINCE (1990), «Aid for small exporting firms : the role of government and information networks », Halifax, Institut de recherches politiques.

Christensen, P.R. (1991), «The small and medium-sized exporter' squeeze : empirical evidence and model reflections», Entrepreneurship \& Regional Development, vol. 3, p. $49-65$.

DENIS, J.E. et D. DÉPELTEAU (1985), « Market knowledge, diversification and expansion », Journal of International Business Studies, vol. 7, p. 77-89.

EVIRGEN, C. et al. (1993), «Information needs of exporters : an empirical study of Turkish exporters », Marketing Intelligent \& Planning, vol. 11, $\mathrm{n}^{\circ}$ 2, p. 28-36.

GIBIAT, M. (1993), «Les modèles de la décision d'exporter en PME/PMI : synthèse des recherches depuis 20 ans », Revue internationale P.M.E., vol. 7, $\mathrm{n}^{\circ}$ 2, p. 11-30.

GofFetTE-NAGOT, N. F. et B. SCHMiTT (1998), «Les proximités, la ville et le rural », dans J.M. Huriot (éd.), La ville ou la proximité organisée, Paris, Anthropos, Collection «Villes », p. 173-183.

JAYET, H. (1996), «Peut-on caractériser les espaces ruraux par l'abondance d'espace?», Revue d'économie régionale et urbaine, $\mathrm{n}^{\mathrm{o}} 2$ 2, p. 201-210.

JULIEN, P.A. (1995), «New technologies and technological information in small business », Journal of Business Venturing, vol. 10, $\mathrm{n}^{\circ} 6$, p. 459-476. 
JULIEN, P.A. (1996), «Globalization: differents types of small business behaviour», Entrepreneurship and Regional Development, vol. 3, $\mathrm{n}^{\circ}$ 1, p. 57-74.

JULIEN, P.A. et M. MORIN (1996), Mondialisation de l'économie et PME québécoises, Sainte-Foy, Presses de l'Université du Québec.

Julien, P.A, J.M. Toulouse, C. Ramangahaly et M. Morin (1998), « Comportements d'information, compétitivité et performance des PME exportatrices », Communication présentée au $4^{\mathrm{e}}$ Congrès international francophone de la PME, Metz, octobre.

LÉO, P.Y. (1993), «Le développement international des PME-PMI, outils pour une analyse stratégique », Communication au Colloque PME-PMI, développement international, CER, Université Aix-Marseille III, juin.

MARCotTe, C. et P.A. Julien (1995), «Partage d'information et performance des coentreprises implantées par les PME québécoises dans les pays en développement », Revue internationale P.M.E., vol. 8, $\mathrm{n}^{\circ}$ 2, p. 175-202.

MADSEN, T.K. (1987), «Empirical export performance studies », Advances in International Marketing, vol. 2, p. 178-198.

MÉRENNE-SCHOUMAKER, B. (1991), La localisation des industries, Paris, Nathan Université.

OCDE (1995), PME et mondialisation, Paris.

ProulX, P.P. (1991), «Cadre conceptuel et éléments théoriques pour l'analyse de la localisation des activités économiques : le cas des villes internationales», Revue Canadienne des Sciences Régionales, vol. 14, $\mathrm{n}^{\circ}$ 2, p. 257-274.

POLÈSE, M. (1994), Économie urbaine et régionale : logique spatiale et mutations économiques, Paris, Economica.

Pyke, F. et W. Sengerberger (éd.)(1990), Industrial Districts and Local Economic Regeneration, Genève, Institut international d'études sociales, BIT.

SAVOIE, D.J. (1993), Mondialisation et gestion publique, Ottawa, Université d'Ottawa, Centre canadien de gestion.

SERINGHAUS, R. (1987), «The role of information assistance in the small firm's export involvement », International Small Business Journal, vol. 5, $\mathrm{n}^{\circ}$ 2, p. 26-36.

SERINGHAUS, R. (1993), «Comparative marketing behavior of Canadian and Austrian hightech exporters», Management International Review, vol. 11, p. 17-27.

SAMIEE, S. et P.G.P. WALTERS (1990), «Influence of firms size on export planning and performance», Journal of Business Research, vol.10, $\mathrm{n}^{\circ}$ 3, p. 5-25.

TELLIER, L.N. (1993), Économie spatiale, réalité économique de l'espace habilité, $2^{\mathrm{e}}$ édition, Boucherville, Gaëtan Morin Éditeur. 